\title{
Special Issue on: Towards a "Smart Society" Through Digital and Wireless Communication Technology
}

\author{
Punnarumol Temdee ${ }^{1}$. Chayapol Kamyod ${ }^{1} \cdot$ Yahoui Hamed $^{2} \cdot$ Uma S. Jha $^{3}$. \\ Minoru Okada ${ }^{4}$
}

Published online: 25 November 2020

(c) Springer Science+Business Media, LLC, part of Springer Nature 2020

This special issue of wireless personal communication focuses on digital and wireless communication technology promoting a smart society. A smart society is the use of technology to shape a better world. It employs the potential digital technology, connected devices, and communication networks to improve people's lives. Simultaneously, it can be considered as an empowered society where human beings and emerging technologies are seamlessly connected. The emergence of multiple contexts and emerging technologies have resulted in new applications and services over a heterogeneous network. This special issue aims to present recent advances and challenges in different aspects relating to constructing a smart society covering from the principle knowledge, infrastructures, and applications. The initial goal is to extend some selected papers from the 6th Global Wireless Summit (GWS 2018) and the 21st International Symposium on Wireless Personal Multimedia Communications (WPMC2018). However, it is also open for the original and unpublished contributions solicited in relevant areas of the principle knowledge, infrastructure, technologies, and applications of digital and wireless communication technology for a smart society.

The contributions in this special issue can be classified into two groups. Firstly, the works relating to principle knowledge, infrastructure, and technologies are demonstrated

Punnarumol Temdee

punnarumol@mfu.ac.th

Chayapol Kamyod

chayapol.kam@mfu.ac.th

Yahoui Hamed

hamed.yahoui@univ-lyon1.fr

Uma S. Jha

Uma.s.jha@raytheon.com

Minoru Okada

mokada@is.naist.jp

1 Computer and Communication Engineering for Capacity Building Research Center, School of Information Technology, Mae Fah Luang University, Chiang Rai, Thailand

2 Faculté des Sciences et Technologies, Université Claude Bernard Lyon 1, Villeurbanne, France

3 Raytheon, Space and Airborne Systems, RF Systems, El Segundo, CA, USA

4 Nara Institute of Science and Technology, Division of Information Science, 8916-5 Takayama, Ikoma, Nara 630-0101, Japan 
for promoting the next generation of wireless communication and applications. Secondly, the smart applications-oriented works for the smart society are illustrated. The findings in this special issue have confirmed that digital and wireless technology's emerging contribution is widely spread in many other disciplines, such as education, healthcare, business, and agriculture. Additionally, these pieces of evidence can guarantee that a smart society's demand is approaching and appealing. The global awareness of smart innovations over a heterogeneous network is called out, requiring the long-term cross-collaboration among academics, research, and industry to satisfy the urgent need of incoming smart society.

Publisher's Note Springer Nature remains neutral with regard to jurisdictional claims in published maps and institutional affiliations.
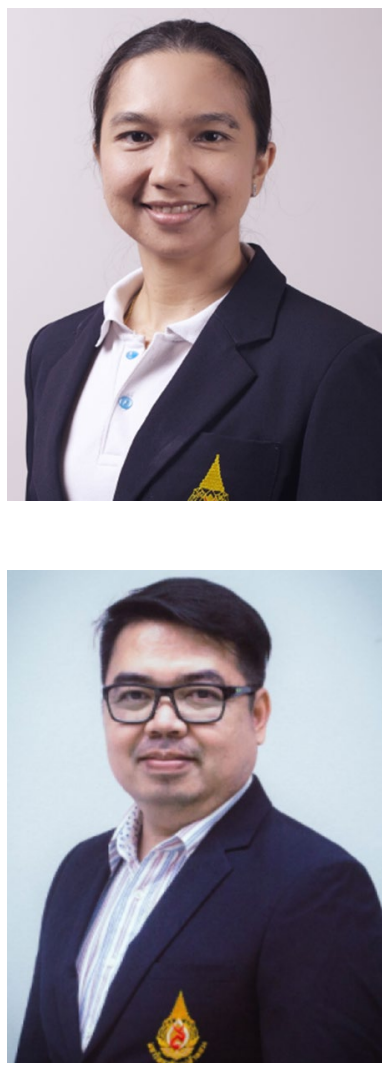

Assoc. Prof. Dr. Punnarumol Temdee received B.Eng. in Electronic and Telecommunication Engineering, M. Eng. in Electrical Engineering, and Ph.D. in Electrical and Computer Engineering from King Mongkut's University of Technology Thonburi. She is currently a lecturer at School of Information Technology, Mae Fah Luang University, Chiang Rai, Thailand. Her research interests are social network analysis, artificial intelligence, software agent, context-aware computing, and ubiquitous computing.

Dr. Chayapol Kamyod received his Ph.D. in Wireless Communication from the Center of TeleInFrastruktur (CTIF) at Aalborg University (AAU), Denmark. He received M. Eng. in Electrical Engineering from The City College of New York, New York, USA. In addition, he received B.Eng. in Telecommunication Engineering and M. Sci. in Laser Technology and Photonics from Suranaree University of Technology, Nakhon Ratchasima, Thailand. He is currently a lecturer in Computer Engineering program at School of Information Technology, Mae Fah Luang University, Chiang Rai, Thailand. His research interests are resilience and reliability of computer network and system, wireless sensor networks, embedded technology, and IoT applications. 


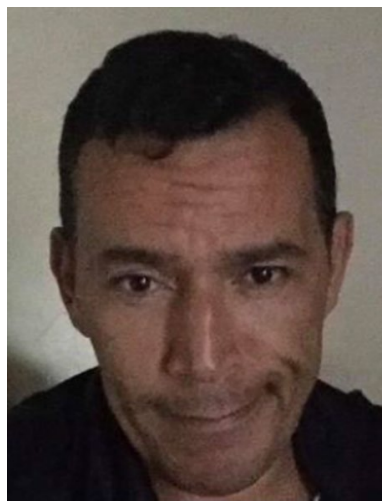

Dr. Yahoui Hamed is an Electrical researcher working in the AMPERE Laboratory of the university of Lyon 1 in Lyon, France. He is currently the head of an international master in advanced manufactured system and Electrical Energy management with more than 80 students having either an international profile or a lifelong learning profile (part-time worker). His research lines focused on electrical diagnostic method and the development of DC microgrids with their corresponding protection and communication system. He has contributed and managed several National and European projects (EIE-SURVEYOR, ELLEIEC, GLINK, ANR C3u). He is author of more than 100 publications.

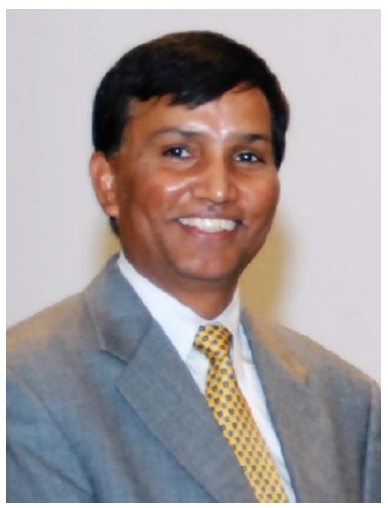

Dr. Uma S. Jha is a Senior Fellow at L3Harris Technologies. Prior to L3Hais he served as an Engineering Fellow at Raytheon, Technical Fellow at Boeing, Director at Qualcomm and many other fortune5 00 companies, as well as high profile start-ups in various executive roles. As a Director of Product Management at Qualcomm, a fabless semiconductor company specializing in wireless SOC development, he managed multibillion-dollar chip business spanning tier-1 OEM accounts globally. At Morhics, a startup in Silicon Valley, he led the development of waveform agnostic signal processing core, a key technology enabler for multimode/multiband smartphone for cellular industry. The company got acquired by Infineon and the IP developed by Dr. Jha's team was used in 1st Apple iPhone, which changed the cellular landscape forever. Dr. Jha has $20+(15$ already granted) patents in consumer electronics domain and actively participates at industry consortia, standards fora, and academia as a keynote speaker, panelist, and advisor. He has published more than 30 papers and coauthored a book on next generation broadband communication systems. He is a Sr. Member of IEEE and serves as Technical Program Committee (TPC) on many IEEE and International conferences. He has organized an international conference, WPMC 2006, as the General Chair where more than 600 people participated form 43 countries. Dr. Jha received a joint Ph.D. from University of Southern California, Los Angeles, California, a MSEE from California State University, and BSEE from BIT Sindri. Additionally, he received an Engineering Management certification from California Institute of Technology, USA.

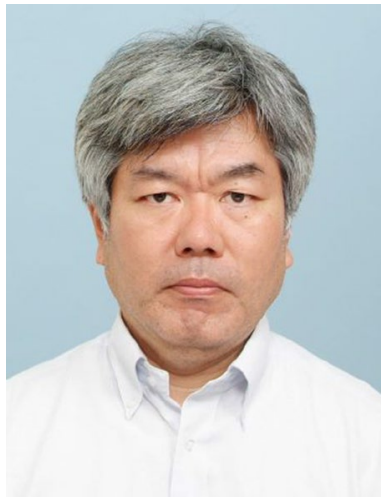

Dr. Minoru Okada received the B.E. degree from the University of Electro-Communications, Tokyo, Japan, in 1990, the M.E. and Ph.D. degrees from Osaka University, Osaka, Japan, in 1992 and 1998, respectively, all in communications engineering. In 2000, he joined Nara Institute of Science and Technology, Nara, Japan, as an Associate Professor and became a Professor in 2006. His research interest is wireless communications. 